\title{
Natural T cell autoreactivity to melanoma antigens: clonally expanded melanoma-antigen specific CD8 + memory T cells can be detected in healthy humans
}

\author{
Anna Przybyla ${ }^{1,2}$ (1) Ting Zhang ${ }^{1} \cdot$ Ruliang Li $^{1} \cdot$ Diana R. Roen ${ }^{1} \cdot$ Andrzej Mackiewicz ${ }^{2,3} \cdot$ Paul V. Lehmann ${ }^{1}$
}

Received: 9 April 2018 / Accepted: 24 December 2018 / Published online: 19 February 2019

(C) The Author(s) 2019

\begin{abstract}
We used four-color ImmunoSpot ${ }^{\circledR}$ assays, in conjunction with peptide pools that cover the sequence of tyrosinase (Tyr), melanoma-associated antigen A3 (MAGE-A3), melanocyte antigen/melanoma antigen recognized by T cells 1 (Melan-A/ MART-1), glycoprotein 100 (gp100), and New York esophageal squamous cell carcinoma-1 (NY-ESO-1) to characterize the melanoma antigen (MA)-specific CD8 + cell repertoire in PBMC of 40 healthy human donors (HD). Tyr triggered interferon gamma (IFN- $\gamma$ )-secreting CD8 + T cells in $25 \%$ of $\mathrm{HD}$ within $24 \mathrm{~h}$ of antigen stimulation ex vivo. MAGE-A3, Melan-A/MART-1, and gp100 also induced recall responses in 10\%, 7.5\%, and 2.5\% of HD, respectively. At this time point, these CD8 + T cells did not yet produce GzB (granzyme B). However, they engaged in GzB production after $72 \mathrm{~h}$ of antigen stimulation. By this 72-h time point, 57.5\% of the HD responded to at least one, and typically several, of the MA. A closer characterization of the Tyr-specific CD8 + T cell repertoire indicated that it was low-affinity, and to primarily entail a stem cell-like subpopulation. Collectively, our data reveal pre-existing endogenous $\mathrm{T}$ cell immunity against melanoma antigens in healthy donors, and analogous to natural autoantibodies, we have termed this "natural T cell autoreactivity".
\end{abstract}

Keywords CD8 + T cells $\cdot$ Melanoma antigens $\cdot$ ELISPOT $\cdot$ Stem-cell like CD8 + T cells $\cdot$ PIVAC 17

This Original Article is based on a presentation given at the Seventeenth International Conference on Progress in Vaccination against Cancer (PIVAC 17), held in Loutraki, Corinthia, Greece, 27th-30th September, 2017. It is part of a Cancer Immunology, Immunotherapy series of PIVAC 17 papers.

Note on previous publication: Parts of this paper including Figs. 1-5 were published before as a poster at http://www.immun ospot.com. Figure 3 was included in a poster presented during the 10th Anniversary International Conference of Contemporary Oncology "Progress in Cancer Research and Therapy". Poznan, 21-23 March 2018.

Electronic supplementary material The online version of this article (https://doi.org/10.1007/s00262-018-02292-7) contains supplementary material, which is available to authorized users.

Paul V. Lehmann

paul.lehmann@immunospot.com

1 Research and Development Department, Cellular Technology Limited (CTL), 20521 Chagrin Boulevard, Shaker Heights, Cleveland, OH 44122-5350, USA

$\begin{array}{ll}\begin{array}{l}\text { Abbreviations } \\ \text { CEFpp32 }\end{array} & \begin{array}{l}\text { Cytomegalovirus-, Epstein-Barr virus } \\ \text { and Flu virus pool of 32 peptides } \\ \text { Cellular Technology Limited }\end{array} \\ \text { CTL } & \begin{array}{l}\text { Glycoprotein 100 } \\ \text { gp100 }\end{array} \\ \text { GzB } & \text { Heanzyme B } \\ \text { HD } & \text { Interferon gamma } \\ \text { IFN- } \gamma & \text { Interleukin 2 } \\ \text { IL-2 } & \text { Melanoma antigen } \\ \text { MA } & \text { Melanoma-associated antigen A3 } \\ \text { MAGE-A3 } & \text { Melanocyte antigen/melanoma antigen } \\ \text { Melan-A/Mart-1 } & \text { recognized by T cells 1 } \\ \text { NY-ESO-1 } & \text { New York esophageal squamous cell } \\ \text { carcinoma-1 } \\ \text { SC } & \text { Stem-cell like T cells }\end{array}$

2 Department of Cancer Immunology, Chair of Medical Biotechnology, Poznan University of Medical Sciences, Poznan, Poland

3 Department of Diagnostics and Cancer Immunology, Greater Poland Cancer Centre, Poznan, Poland 


$\begin{array}{ll}\text { SFU } & \text { Spot forming unit } \\ \text { TNF- } \alpha & \text { Tumor necrosis factor alpha } \\ \text { Tyr } & \text { Tyrosinase }\end{array}$

\section{Introduction}

Successful immune therapeutic approaches to melanoma [1] have drawn attention to the importance of monitoring melanoma antigen- (MA-) specific T cells. Melanoma is considered a highly immunogenic tumor $[2,3]$ with a $\mathrm{T}$ cell response to the tumor spontaneously developing as soon as it metastasizes into the draining lymph nodes [4]. It is thought that prior to this priming event, the MA-specific $\mathrm{T}$ cells are antigen-inexperienced/naïve [4] and like the naïve antigenspecific $\mathrm{T}$ cell repertoires in general, occur in frequencies far too low for detection. However, the opportunity to characterize MA-specific $T$ cells increases after these $T$ cells have been primed, clonally expanded, and differentiated [5]. For this reason, most successful efforts to characterize MA-specific T cells have focused on melanoma patients in whom the metastasizing tumor has already primed a $\mathrm{T}$ cell response. Consequently, there is relatively little information available on the MA-specific repertoire before this priming event, that is, in healthy subjects. This report aims at filling this gap. By characterizing the MA-specific T cell repertoire in healthy donors, we aim at establishing the baseline against which spontaneously-developing and experimentally induced immunity to melanoma can be compared.

The initial efforts to characterize MA-specific T cells not only focused on patients with metastasizing melanoma, but also involved protocols that aimed at expanding MA-specific $\mathrm{T}$ cells in vitro for several weeks before analysis. Frequently, these protocols involving repeated antigen stimulation cycles in the presence of growth factors [5, 6]. As the limit of detection using flow cytometry is roughly one antigen-specific $\mathrm{T}$ cell within 10,000 bystander cells (that is, $0.01 \%$, [7]), this frequently used amplification step aims to enable detection of rare MA-specific $\mathrm{T}$ cells that would otherwise go undetected [5]. More importantly, CD8 + T cell subpopulations do not possess equivalent expansion rates [8,9], and can alter their activation/differentiation status after antigen stimulation depending upon the culture conditions [10]. Therefore, concerned by altering $\mathrm{T}$ cell repertoires through in vitro culture, the field is moving to the analysis of $\mathrm{T}$ cells freshly isolated from blood, that is, ex vivo. In the present study, we performed ex vivo analysis of MA-specific T cells.

Enzyme-linked immunospot assay (ELISPOT) analysis has been gaining increasing popularity for ex vivo immune monitoring [11]. Unlike the multimer approach [12], ELISPOT assays are not limited to single peptides that are tailored to individual HLA alleles. Instead, extensive peptide libraries can be tested simultaneously as pools to systematically accommodate the array of potential antigenic determinants, irrespective of the test subject's HLA type. Relatively little information is lost through pooling peptides since the numbers of cells recognizing the individual peptides contained in the pool add up to the number of cells activated by the pooled peptides [13].

For a long time, a weakness of ELISPOT assays $v s$. multimer analysis was that ELISPOT was suited only for single color/parameter analysis. Namely, counting the numbers of antigen-stimulated $\mathrm{T}$ cells in the test sample that secrete one analyte at a time, typically interferon-gamma (IFN- $\gamma$ ). In contrast, multimer-stained cells can be counterstained for expression of cell surface markers. Such multimer studies revealed that $\mathrm{CD} 8+\mathrm{T}$ cells occur in subpopulations with fundamentally different surface phenotypes, translating into altered characteristics and contributions to host defense (summarized for cancer-relevant CD8 $+\mathrm{T}$ cell immunity in [8]). Ongoing efforts to characterize antigen-specific $\mathrm{CD} 8+\mathrm{T}$ cells primarily focus on defining their surface phenotype, and in turn, seek to detail their activation/differentiation status. In the present study, we rely on a novel four-color ImmunoSpot ${ }^{\circledR}$ analysis approach to similarly define the effector capabilities of antigen-specific $\mathrm{T}$ cell subpopulations [14].

In the present study, we leverage the strength of ELISPOT to analyze the MA-specific T cell repertoire from healthy human donors (HD) directly ex vivo. First, we take advantage of the high sensitivity of ELISPOT. As performed, our assays are capable of enumerating individual MA antigen-specific CD8 + T cells within 250,000 PBMC [15]; corresponding to a detection limit of $0.003 \%$. Second, we leverage the capacity of ELISPOT for assays using large peptide libraries. In this study, we utilize peptide libraries representing MA antigens, such as tyrosinase (Tyr), melanoma-associated antigen A3 (MAGE-A3), melanocyte antigen/melanoma antigen recognized by $\mathrm{T}$ cells 1 (Melan-A/ MART-1), glycoprotein 100 (gp100), and New York esophageal squamous cell carcinoma-1 (NY-ESO-1). Each antigen is represented by an individual peptide library that systematically covers the entire amino acid sequence. All potential antigenic determinants of MA antigens are thus presented, irrespective of the HLA type of the donor and which HLA allele(s) serve as the restriction element(s). Third, we perform four-color ELISPOT assays to simultaneously measure IFN- $\gamma$, tumor necrosis factor alpha (TNF- $\alpha$ ), IL-2, and GzB production. Collectively, the cytokine secretion profile of individual MA-specific CD8 + T cells defines their subpopulation lineage [14]. Finally, we measure the affinity [16] of MA-specific CD8 + T cells. By providing a comprehensive, high-resolution analysis of the MA-specific CD8 + T cell repertoire in HD, we introduce the feasibility of this approach and establish the baseline for immune monitoring of unvaccinated or vaccinated melanoma patients. 


\section{Materials and methods}

\section{PBMC donors}

Peripheral blood mononuclear cells (PBMC) from healthy human donors (HD) were selected from the ePBMC library [Cellular Technology Limited (CTL), Shaker Heights, OH, USA]. The characteristics of the selected donors are specified in Suppl. Table 1. The PBMC were stored in the vapor phase of liquid nitrogen until testing. Processing (thawing, washing, and counting) of PBMC was done according to CTL's protocols [17]. The viability of thawed cells exceeded $90 \%$ for all PBMC samples. The PBMC were resuspended at a final concentration of $2.5 \times 10^{6} \mathrm{PBMC} / \mathrm{ml}$ in CTL-Test Medium (CTLT-005, from CTL) of which $100 \mu \mathrm{l}(250,000$ cells) was plated per well into ELISPOT assays.

\section{Antigens}

Five melanoma-associated antigens were used for this study, all were purchased from JPT (Berlin, Germany): PepMix Human (tyrosinase)-PM-Tyr; PepMix Human (MAGEA3)—PM-MAGEA3; PepMix Human (Melan-A/ Mart-1)_PM-MelA; PepMix (Melanocyte protein Pmel 17gp100)—PM-GP100; PepMix (NY-ESO-1)—PM-NYE. Peptide pools consisted of 15-mer peptides that systematically cover the entire amino acid (aa) sequence of the respective protein in steps of 11 aa. All peptide pools were tested at a final concentration of $1 \mu \mathrm{g} / \mathrm{ml}$ for each peptide, with all peptides within the pool being represented at equimolar ratios. CEFpp32 (Cytomegalovirus-, Epstein-Barr virus and Flu virus pool of 32 peptides) was used as a positive control for $\mathrm{CD} 8+\mathrm{T}$ cell reactivity [18]. This peptide pool was derived from CTL (Cat \#: CTL-CEF-002) and used at a final concentration of $0.25 \mu \mathrm{g} / \mathrm{ml}$.

\section{Human single color IFN- $\gamma$ and granzyme B ImmunoSpot ${ }^{\circledR}$ assays}

Single-color enzymatic ELISPOT assays were performed to detect PBMC secreting IFN- $\gamma$ (CTL, Cat \#: HIFNG-1/5M) or granzyme B (CTL, huGzB) following the manufacturer's recommendations. These two single color enzymatic assays were performed in an identical manner, except for the use of the respective capture and detection antibodies. Briefly, in the first step, the PVDF membrane was pre-coated with capture antibody overnight. Next, antigens were dissolved in CTL-Test Medium (CTL, Cat \# CTLT-005) and plated in $100 \mu \mathrm{l}$ per well. CTL-Test Medium and PBMC alone constituted the negative control wells, with CEFpp32 and CPI as positive controls [19]. The plates with the antigen were stored at $37{ }^{\circ} \mathrm{C}$ in a $\mathrm{CO}_{2}$ incubator until the cells were ready for plating. PBMC were added at 250,000 cells/well in $100 \mu \mathrm{l}$ using wide-bore pipette tips. Plates were gently tapped on each side ensuring even distribution of the PBMC in the wells. The cells were incubated with the antigens for $24 \mathrm{~h}$ or $72 \mathrm{~h}$, as specified, in a humidified incubator at $37^{\circ} \mathrm{C}$ and $9 \% \mathrm{CO}_{2}$. After the incubation time, cells were removed, detection antibody added, and enzymatic visualization of the plate-bound cytokine performed by enzyme-catalyzed substrate precipitation. The plates were air-dried prior to analysis. ELISPOT plates were analyzed using an ImmunoSpot ${ }^{\circledR}$ S6 Ultimate Reader from CTL. Spot Forming Units (SFU) were automatically counted by the ImmunoSpot ${ }^{\circledR}$ Software (CTL) using the Basic Count Suite.

\section{Human four-color ImmunoSpot ${ }^{\circledR}$ assay}

To identify $\mathrm{CD} 8+\mathrm{T}$ cell subpopulations by co-expression profiles of IFN- $\gamma$, GzB, IL- 2 and TNF- $\alpha$, four-color ImmunoSpot ${ }^{\circledR}$ assays [14] were performed (CTL, hT4001F) using PBMC with known IFN- $\gamma$ reactivity from previous experiments. Cells were pre-stimulated for $72 \mathrm{~h}$ with antigen (tyrosinase or CEFpp32) in 24-well plates at $2 \times 10^{6}$ cells/well in an incubator at $37{ }^{\circ} \mathrm{C}, 9 \% \mathrm{CO}_{2}$. These antigens were used at final concentrations of $1 \mu \mathrm{g} / \mathrm{ml}$ and $0.25 \mu \mathrm{g} /$ $\mathrm{ml}$, respectively. Thereafter, the cells were transferred into a 96-well PVDF membrane plate with low auto-fluorescence (contained in the kit) that was pre-coated with the four capture antibodies. The cells were added at $3 \times 10^{5}$ cells/well, along with Tyr or CEFpp32 antigens at the concentration specified above and anti-CD28 $(0.1 \mu \mathrm{g} / \mathrm{ml})$. After $24 \mathrm{~h}$ incubation at $37{ }^{\circ} \mathrm{C}, 9 \% \mathrm{CO}_{2}$, the plates were washed and then incubated for $2 \mathrm{~h}$ at room temperature with the respective detection antibodies: anti-IFN- $\gamma$ (CTLhT02), anti-TNF- $\alpha$ (CTLhT13), anti-IL2 (CTLhT56), and anti-GzB (CTLhT59). The plates were then washed and incubated with the tertiary solutions for $1 \mathrm{~h}$ at room temperature. Detection and tertiary solutions were filtered through a $0.22 \mu \mathrm{m}$ filter prior to usage. Following completion of detection steps, plates were washed, dried, scanned, and analyzed using an ImmunoSpot ${ }^{\circledR}$ S6 Ultimate Analyzer.

\section{CD8 + and CD4 + T cell depletion}

To establish whether the peptide-specific $\mathrm{T}$ cell response was present in the $\mathrm{CD} 4+$ or $\mathrm{CD} 8+$ lineage, $\mathrm{CD} 4+$ or $\mathrm{CD} 8+$ cells were depleted from PBMC using magnetic beads (Stemcell Technologies Inc, Canada). The depletion assay was performed according to manufacturer's instructions. Unseparated PBMC, or CD4 + or CD8 + cell-depleted populations were then plated on 96 -well ELISPOT plates at $2.5 \times 10^{5}$ cells/well, and the assay performed as described previously. 


\section{Statistical analysis}

ELISPOT counts follow Normal distribution among replicate wells, which permits the utilization of parametric statistics for identifying positive responses [20]. Accordingly, the Student's $t$ test was used for comparing SFU in the three antigen-containing replicate wells vs. the spot counts in the medium control wells. A $p$ value $<0.05$ was considered as the cut-off for positivity, with an additional requirement that spot counts in antigen-stimulated wells exceed $10 \mathrm{SFU} /$ well.

\section{Results}

\section{Detection of melanoma antigen-specific $T$ cells secreting IF $\mathrm{N}-\gamma$ in healthy human donors}

PBMC of 40 healthy adult donors (HD) were randomly selected from CTL's ePBMC library. The age, gender, and HLA-type of these donors are listed in Suppl. Table 1. Five melanoma antigens (MA) antigens were used for evaluating donor cell reactivity. Each of these antigens was represented by a pool of 15-mer overlapping peptides that cover the entire amino acid (aa) sequence of the respective proteins. Tyrosinase (Tyr), a protein of 529 aa was represented by 117 peptides. Melanocyte protein Pmel 17 gp100, is a protein of 661 aa length that was covered by 163 peptides. Melan-A/MART-1, a protein of 118 aa was covered by 27 peptides. Melanoma-associated antigen MAGE-A3, 314 aa long, was represented by 76 overlapping peptides. The NY-ESO-1 tumor antigen of the cancer/testis family, with a length of 180 aa, was covered by 43 peptides. Each peptide within the respective pool was present in equimolar ratio, and each peptide pool was tested at $1 \mu \mathrm{g} / \mathrm{ml}$ concentration with the PBMC present at 250,000 cells per well. Under these conditions, the number of peptide-triggered IFN- $\gamma$ producing cells is enumerated as spot-forming units (SFU) within the 250,000 PBMC present per well, that is, the frequency of such antigen-specific $\mathrm{T}$ cells is equal to the SFU count $/ 250,000$.

In the first set of experiments, PBMC from $40 \mathrm{HD}$ were exposed to the 5 MA peptide pools for $24 \mathrm{~h}$ or $72 \mathrm{~h}$, during which IFN- $\gamma$ production was measured in a standard ImmunoSpot ${ }^{\circledR}$ assay. In the absence of antigen stimulation, less than 5 cells per well spontaneously produced IFN- $\gamma$ for each of the donors at either the $24 \mathrm{~h}$ or the $72 \mathrm{~h}$ time points. Thus, the medium background was less than $5 \mathrm{SFU} /$ well. To meet the positivity criterion, the difference between the three replicate medium control wells tested and the three replicate wells containing the test MA peptide pool needed to reach a statistical significance of $p<0.05$ in the Student's $t$ test. For increased stringency towards scoring as positive, we made an additional requirement that spot counts in antigenstimulated wells exceed $10 \mathrm{SFU}$ per well.

Figure 1 shows representative examples of tyrosinaseinduced IFN- $\gamma$ spot formation after 24 and $72 \mathrm{~h}$ antigen stimulation. In some donors, for whom HD \#7 is shown in this figure as an example, the tyrosinase peptide pool stimulated IFN- $\gamma$ producing cells within $24 \mathrm{~h}$. Nine of the 40 HD tested $(22.5 \%)$ fell in this category (Table 1). Of note, in these donors the number of Tyrosinase-specific $\mathrm{T}$ cells capable of IFN- $\gamma$ secretion was not substantially increased at the $72 \mathrm{~h}$ time point. Likewise, the number of CEF-triggered SFU did not differ much when measured at the 24 or $72 \mathrm{~h}$ time points (Fig. 1; Table 1). Moreover, the quality of such tyrosinase-induced spots (reflecting on the amount of IFN- $\gamma$ secreted by the individual T cells) was similar to the spots triggered by the CEF peptide pool positive control. Strikingly, the numbers of spots induced by the tyrosinase peptide pool frequently reached the magnitude of the response triggered by the CEF pool. Thus, the frequency of tyrosinase-reactive $T$ cells in the blood was frequently as high, or within an order of magnitude, as the CEF antigen-reactive repertoire. Therefore,
Fig. 1 IFN- $\gamma$ ELISPOTS induced by tyrosinase in PBMC of healthy donors (HD) within 24 and $72 \mathrm{~h}$ of antigen stimulation. Representative well images are shown for the data summarized in Table 1 - the legend to Table 1 applies

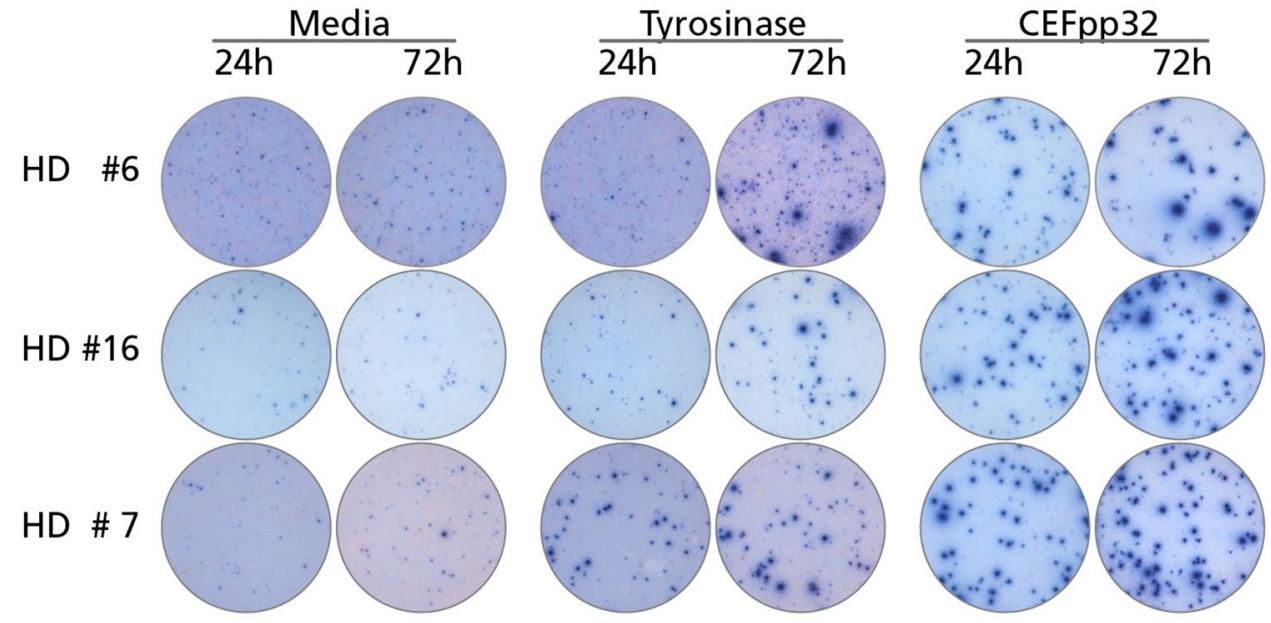


Table 1 Melanoma antigentriggered IFN- $\gamma$ spot forming units in PBMC of healthy donors

\begin{tabular}{|c|c|c|c|c|c|c|}
\hline Donor no * & Tyr & MAGE-A3 & Melan-A/Mart-1 & gp100 & NY-ESO-1 & CEFpp32 \\
\hline 2 & $-/-$ & $4 / 32$ & $-/-$ & $-/-$ & $-/-$ & $7 / \mathbf{1 1}$ \\
\hline 3 & $7 / 22$ & $-/-$ & $-/-$ & $-/-$ & $-/-$ & $197 / 255$ \\
\hline 4 & $10 / \mathbf{1 7}$ & $-/-$ & $-/-$ & $-/-$ & $-/-$ & $17 / 22$ \\
\hline 6 & $3 / 22$ & $5 / 16$ & $10 / \mathbf{1 3}$ & $5 / 16$ & $7 / 13$ & $80 / 79$ \\
\hline 7 & $51 / 72$ & $-/-$ & $-/-$ & $-/-$ & $-/-$ & $130 / \mathbf{1 7 0}$ \\
\hline 9 & $32 / 42$ & $2 / 13$ & $-/-$ & $2 / \mathbf{1 3}$ & $-/-$ & not tested* \\
\hline 10 & $-/-$ & $1 / 13$ & $-/-$ & $-/-$ & $-/-$ & not tested* \\
\hline 11 & $17 / 20$ & $4 / 14$ & $5 / 20$ & $5 / \mathbf{1 3}$ & $3 / \mathbf{1 1}$ & not tested* \\
\hline 13 & $-/-$ & $3 / 12$ & $-/-$ & $-/-$ & $-/-$ & $64 / 86$ \\
\hline 14 & $-/-$ & $5 / \mathbf{1 1}$ & $-/-$ & $-/-$ & $-/-$ & $9 / 9$ \\
\hline 15 & $-/-$ & $1 / 18$ & $-/-$ & $-/-$ & $-/-$ & $15 / 22$ \\
\hline 16 & $12 / 30$ & $76 / 247$ & $2 / \mathbf{1 1}$ & $-/-$ & $-/-$ & $88 / \mathbf{1 4 0}$ \\
\hline 17 & $38 / 46$ & $-/-$ & $-/-$ & $-/-$ & $-/-$ & $86 / 78$ \\
\hline 19 & $-/-$ & $3 / 14$ & $-/-$ & $-/-$ & $-/-$ & $38 / 41$ \\
\hline 20 & $-/-$ & $15 / 19$ & $-/-$ & $-/-$ & $-/-$ & $5 / 4$ \\
\hline 21 & $27 / 31$ & $-/-$ & $-/-$ & $-/-$ & $-/-$ & $301 / 346$ \\
\hline 23 & $-/-$ & $-/-$ & $2 / \mathbf{1 3}$ & $-/-$ & $-/-$ & $3 / 6$ \\
\hline 24 & $7 / 16$ & $31 / 25$ & $10 / 22$ & $8 / 15$ & $4 / 17$ & $125 / 155$ \\
\hline 26 & $16 / 24$ & $-/-$ & $-/-$ & $-/-$ & $-/-$ & 215/189 \\
\hline 27 & $-/-$ & $3 / 18$ & $6 / 11$ & $-/-$ & $-/-$ & $5 / 12$ \\
\hline 31 & $3 / 21$ & $27 / 63$ & $11 / 65$ & $24 / 31$ & $-/-$ & $40 / 48$ \\
\hline 32 & $14 / 17$ & $-/-$ & $-/-$ & $-/-$ & $-/-$ & $87 / \mathbf{1 4 5}$ \\
\hline 36 & $39 / 53$ & $-/-$ & $-/-$ & $1 / 14$ & $-/-$ & 122/166 \\
\hline
\end{tabular}

PBMC (250,000 per well) of the 40 healthy donors specified in Suppl. Table 1 were exposed to the specified MA or the CEFpp32

Donors no*-only those donors who respond to at least one melanoma antigen

Not tested*-CPI was used as a positive control

A Student's $t$ test was done comparing SFU counts for the three antigen-induced replicates with the three replicates of the corresponding medium control. The table shows results for those donors only who scored at least one positive response. The first number represents the SFU counts at $24 \mathrm{~h}$, the second number, in bold, at $72 \mathrm{~h}$. SFU counts that did not reach statistical significance are represented by a hyphen tyrosinase-reactive $\mathrm{T}$ cells from these HD appear to be in vivo primed, clonally expanded, and cytokine-differentiated memory $\mathrm{T}$ cells.

In other donors, for whom HD \#6 and HD \#16 are shown as examples in Fig. 1, the tyrosinase-induced production of IFN- $\gamma$ was at or below the limit of detection. However, antigen-elicited IFN- $\gamma$ production became clear cut by the 72-h time point. For tyrosinase, four of the 40 HD (10\%) fell in this category (Table 1). Similar findings were made for the other MA as well: four of the 40 donors (10\%) responded to MAGE-A3 within $24 \mathrm{~h}$ and the number of responsive donors increased to 14 (35\%) by $72 \mathrm{~h}$. In addition, the number of donors responding to Melan-A/ Mart-1, gp100, and NY-ES0-1 increased from 7.5\%, 2.5\% and $0 \%$ at $24 \mathrm{~h}$ to $17.5 \%, 15 \%$ and $7.5 \%$ by $72 \mathrm{~h}$, respectively (Table 1). Thus, after $72 \mathrm{~h}$ ex vivo, $57.5 \%$ of the HD tested exhibited a response to at least one, and typically several of the MA.

\section{Induction of granzyme B-secreting MA-specific T cells within $72 \mathrm{~h}$ ex vivo}

Following $72 \mathrm{~h}$ of MA stimulation, a substantial IFN- $\gamma$ response was observed using PBMC from multiple HD. Based on the number of spots, these responses most likely originate from clonally expanded, cytokine-differentiated memory CD $8+\mathrm{T}$ cells. To verify this notion, we next tested whether MA-specific T cells could secrete granzyme $\mathrm{B}(\mathrm{GzB})$ at the 72-h time point; which is characteristic for effector CD8 + T cells capable of cytolysis $[8,21]$. We, therefore, repeated the experiments above, exactly as preformed previously, but this time measuring GzB secretion by the PBMC. The PBMC of the same $40 \mathrm{HD}$ were exposed to the five MA, again incubating the cells for $24 \mathrm{~h}$ or $72 \mathrm{~h}$, and then the GzB response enumerated. At the 24-h time point, none of the MA peptide pools triggered GzB secretion in PBMC of HD. This also included the MA/HD combinations 
for which an IFN- $\gamma$ response was detected at $24 \mathrm{~h}$. Specifically, HD \#4, \#7, \#9, \#11, \#16, \#17, \#21, \#26, \#32 and \#36 (Table 1) all demonstrated IFN- $\gamma$ production at $24 \mathrm{~h}$ in response to tyrosinase but were negative for $\mathrm{GzB}$ production. However, nearly all IFN- $\gamma$ positive MA/HD combinations in Table 1 also exhibited strong GzB responses at the 72-h time point (Fig. 2a, b). Therefore, within 3 days of antigen stimulation ex vivo, MA-specific T cells convert into effector cells capable of secreting GzB. Moreover, MA-specific responses were observed irrespective of whether detectable IFN- $\gamma$ secreting cells were already present at the 24 -h time point.

\section{In HD the tyrosinase-induced IFN- $\gamma$ SFU are produced by CD8 $+\mathrm{T}$ cells}

We next performed cell separation experiments to establish whether the MA-triggered IFN- $\gamma$ spots originated from CD4 + and/or CD8 + T cells. Unseparated PBMC were tested along with PBMC that were subjected to $\mathrm{CD} 4+$ or $\mathrm{CD} 8+$ cell depletion. A representative experiment is shown in Fig. 3, in which CD8 + cell depletion nearly completely abrogated the response to the CEF peptide pool. Additionally, the magnitude of the CEF peptide response was largely unaffected by $\mathrm{CD} 4+$ cell depletion. Both of these observations are in line with the notion that the CEF responsive population are $\mathrm{CD} 8+\mathrm{T}$ cells. In contrast, $\mathrm{CD} 4+$ cell depletion, but not CD8 + cell depletion, abrogated the recall response triggered by the CPI antigen pool encompassing proteins from cytomegalovirus, parainfluenza and influenza virus. Collectively, the CPI antigen and CEF peptide pools constitute positive controls for $\mathrm{CD} 4+$ and $\mathrm{CD} 8+\mathrm{T}$ cell activation
[19]. For tyrosinase, CD8 + cell depletion reduced SFU numbers in PBMC over $90 \%$ in some instances, whereas CD4 + cell depletion resulted in a smaller, $40 \%$ reduction. Therefore, the tyrosinase reactive IFN- $\gamma$ secreting cells recalled by the 15 -mer peptide pool were predominantly $\mathrm{CD} 8+\mathrm{T}$ cells; however, there was also a significant percentage of $\mathrm{CD} 4+\mathrm{T}$ cells that were responsive to this antigen. While the CD4+/CD8 + cell lineage of the T cells responding to the other $4 \mathrm{MA}$ were not formally evaluated, the induction of GzB after $72 \mathrm{~h}$ (see Fig. 2b) suggests that these responses also entail a major $\mathrm{CD} 8+\mathrm{T}$ cell component.

\section{Characterization of the tyrosinase-reactive CD8 + T cell lineages in HD}

Cytokine co-expression patterns permit segregation of $\mathrm{CD} 8+\mathrm{T}$ cells into sub-lineages [8]. Terminal effector cells (TE) co-express IFN- $\gamma$, TNF- $\alpha$ and GzB, but do not secrete IL-2. Polyfunctional CD8 $+\mathrm{T}$ cells $(\mathrm{PF})$ secrete all four of these cytokine analytes, while stem-cell like (SC) T cells secrete IL-2 in isolation. To determine which of the above $\mathrm{CD} 8+\mathrm{T}$ cell sub-lineages encompassed the tyrosinasespecific response observed in $\mathrm{HD}$, we performed four-color ImmunoSpot ${ }^{\circledR}$ assays studying the co-secretion of IFN $-\gamma$, TNF- $\alpha$, GzB, and IL-2 by individual antigen-stimulated cells [14]. In parallel, CEF peptide pool-reactive CD8 + T cells were tested for comparison and the results are shown in Fig. 4. In five of nine HD tested, the tyrosinase-specific $\mathrm{CD} 8+\mathrm{T}$ cells were predominantly stem cell-like $\mathrm{T}$ cells (SC: $\mathrm{IL}-2^{+} / \mathrm{IFN}-\gamma^{-}, \mathrm{TNF}-\alpha^{-}$and $\mathrm{GzB}^{-}$), and outnumbered the polyfunctional (PF: IL-2 ${ }^{+} / \mathrm{IFN}-\gamma^{+}, \mathrm{TNF}-\alpha^{+}$and $\mathrm{GzB}^{+}$) and terminal effector cells (TE: IL- $2^{-} / \mathrm{IFN}-\gamma^{+}, \mathrm{TNF}-\alpha^{+}$and a

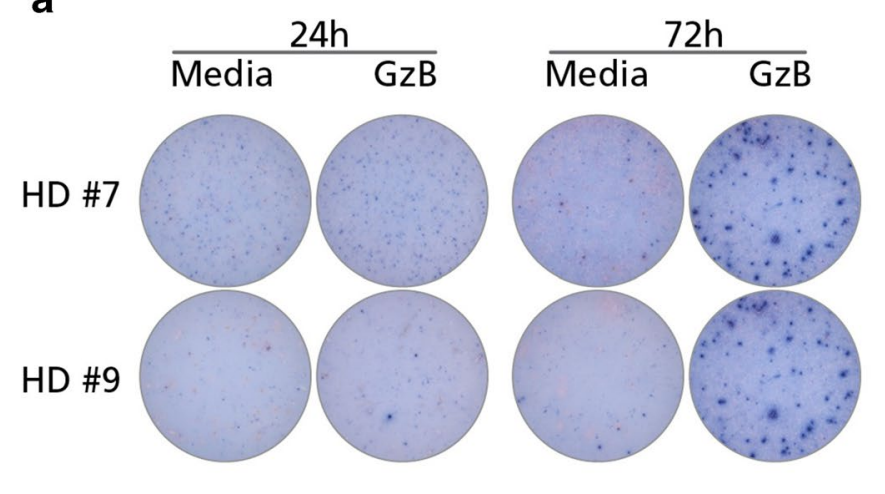

b

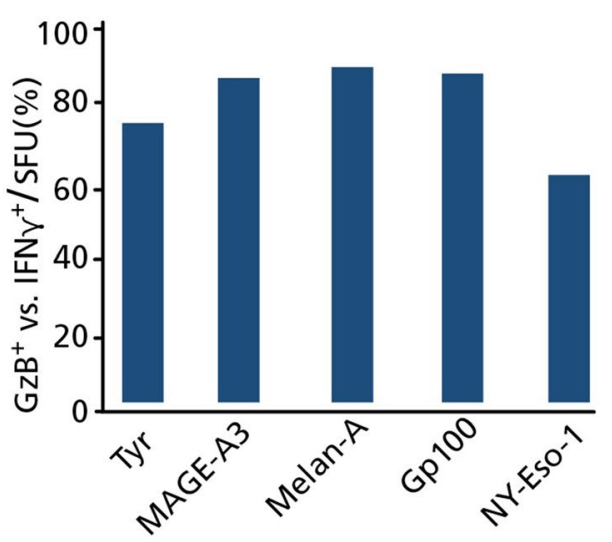

Fig. 2 Granzyme B (GzB) production by PBMC of healthy donors after melanoma antigen exposure. The experiments were performed as detailed in the legend of Table 1 and in the text, except that GzBsecreting cells were detected. a Representative images are shown for two HD who responded with IFN- $\gamma$ production to tyrosinase at the 24-h time point. b GzB positivity of IFN- $\gamma$ positive cultures after $72 \mathrm{~h}$ of MA stimulation, after setting IFN- $\gamma$-positive MA-responses in each $\mathrm{HD}$ as $100 \%$ (see the $72 \mathrm{~h} \mathrm{IFN-} \gamma$ data in Table 1). The percentage of these cultures that were also positive for $\mathrm{GzB}$ at the 72-h time point is depicted in the graph 


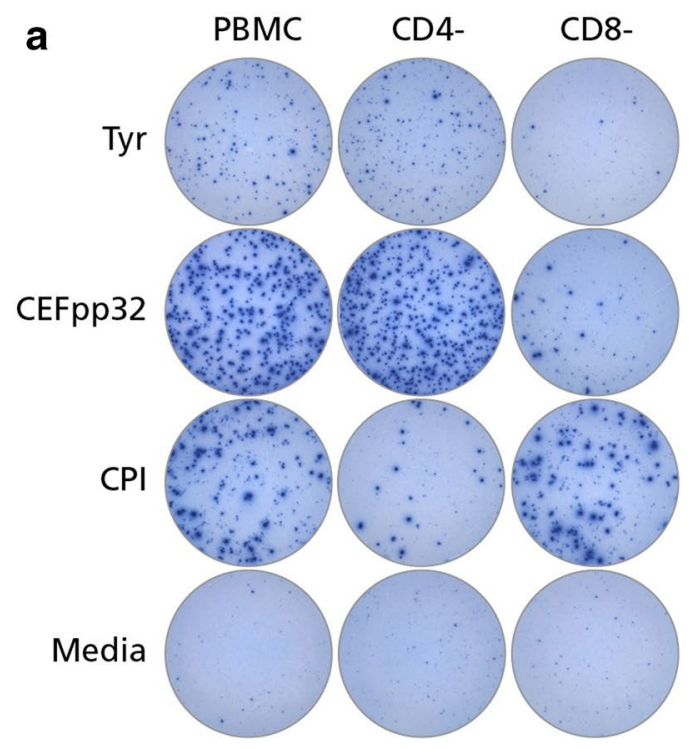

Fig. 3 Tyrosinase-triggered IFN- $\gamma$ spots are produced primarily by CD8 cells. PBMC from HD \#36 were tested "unfractionated", or after depletion of $\mathrm{CD} 4\left(\mathrm{CD}^{-}\right)$or $\mathrm{CD} 8\left(\mathrm{CD} 8^{-}\right)$cells via magnetic beads. These cells were then cultured either with tyrosinase (Tyr), CEF peptides (CEFpp32), CPI, or left unstimulated (media) and IFN- $\gamma$ was b
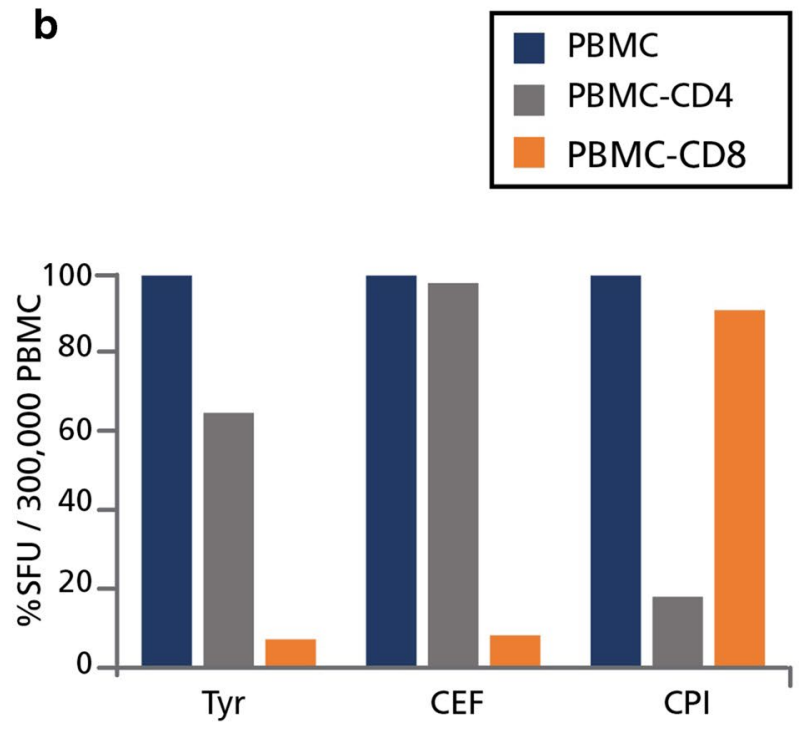

detected after $24 \mathrm{~h}$. a The original wells are shown. b The percentage reduction in spot forming unit (SFU) counts is shown after CD4 or CD8 cell depletion, representing the response of unfractionated PBMC as $100 \%$ a
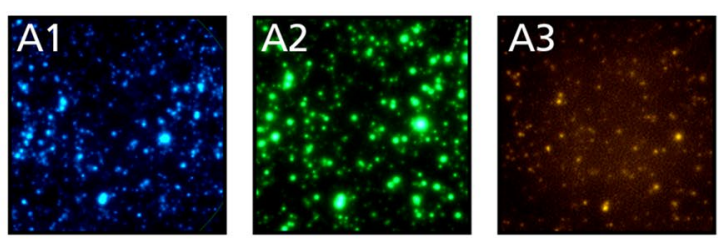

b

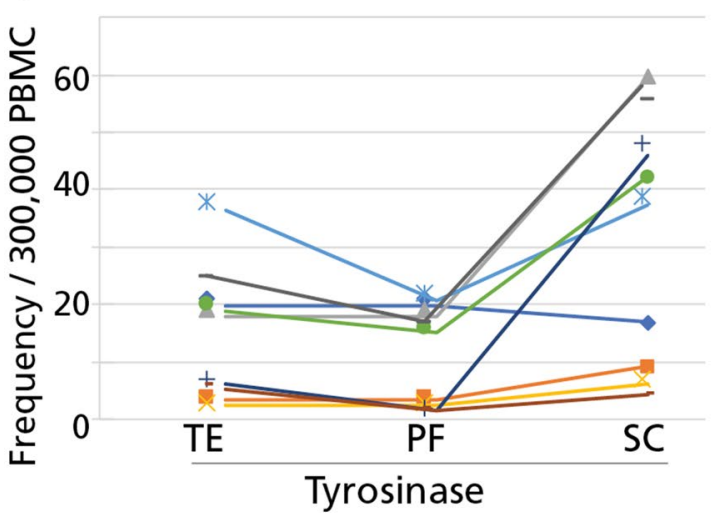

Fig. 4 Lineage characterization of tyrosinase- and CEF peptidereactive CD8 cells of healthy donors. a Representative wells of the individual color planes are shown in A1-A4, for IL-2, IFN- $\gamma$, TNF- $\alpha$, and $\mathrm{GzB}$, respectively. A5 depicts the overlay of the four individual color planes, and polyfunctional cells positive for all four analytes are highlighted in A6. Terminal effector cells (TE) were defined as IL-2-, IFN- $\gamma^{+}, \mathrm{TNF}-\alpha^{+}$, and $\mathrm{GzB}^{+}$; polyfunctional cells (PF) as IL-2 ${ }^{+}$, IFN-
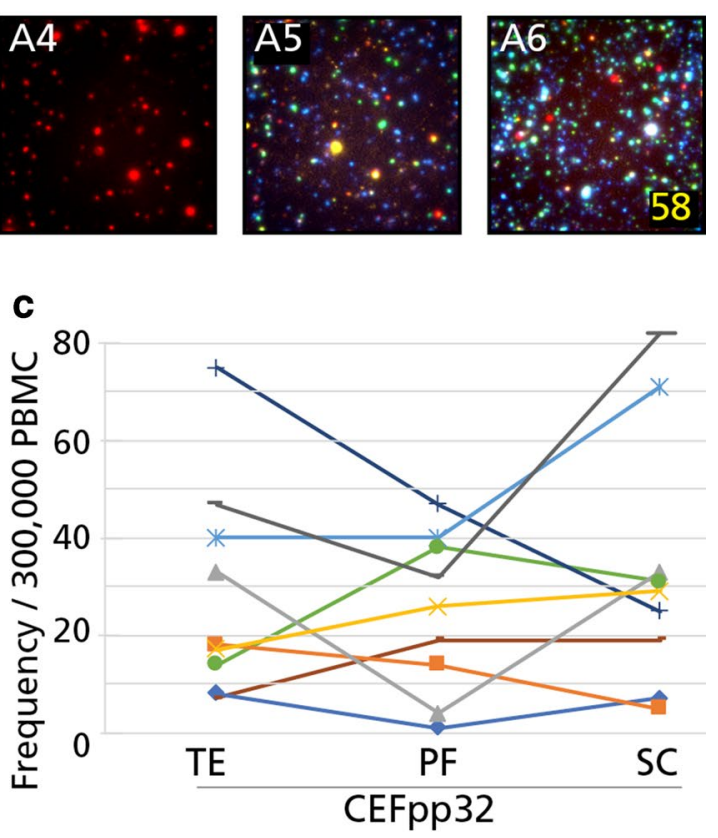

$\gamma^{+}, \mathrm{TNF}-\alpha^{+}$, and $\mathrm{GzB}^{+}$, and stem cell-like cells (SC) as IL-2 ${ }^{+}, \mathrm{IFN}-$ $\gamma^{-}, \mathrm{TNF}-\alpha^{-}$, and $\mathrm{GzB}^{-}$. PBMC of tyrosinase-reactive HD (donor \#2, $\# 7, \# 9, \# 16, \# 17, \# 21, \# 31$ and \#36) were cultured with the tyrosinase (b) or the CEF peptide pool (c) for $72 \mathrm{~h}$, after which they were plated into a four-color ImmunoSpot ${ }^{\circledR}$ assay for $24 \mathrm{~h}$, enabling detection of cells secreting IL-2, IFN- $\gamma$, TNF- $\alpha$, and GzB 
$\mathrm{GzB}^{+}$) by at least twofold. The parallel characterization of CEF-reactive $\mathrm{CD} 8+\mathrm{T}$ cells from these same nine HD showed no bias towards the SC phenotype, and instead PF and TE subpopulations were comparably represented.

\section{Characterization of the affinity of tyrosinase-specific CD8 + T cells in HD}

By titrating the antigenic peptides, one can establish the functional affinity, that is, the threshold for antigen-specific $\mathrm{T}$ cell receptor stimulation. High-affinity T cells respond to lower concentrations of peptide, whereas low-affinity $\mathrm{T}$ cells require higher concentrations of peptide to become activated [16]. Using this approach, we showed, for example, that negative selection in the thymus trims the high-affinity end of the autoantigen-specific $\mathrm{T}$ cell repertoire while sparing $\mathrm{T}$ cells with low affinity for the autoantigen [16]. To establish the functional affinity of the tyrosinase-specific $\mathrm{CD} 8+\mathrm{T}$ cells in HD, donors displaying an IFN- $\gamma$ response within $24 \mathrm{~h}$ were re-tested in an IFN- $\gamma$ assay using titrated quantities of the tyrosinase peptide pool. Response curves to the tyrosinase and CEF peptide pools are depicted in Fig. 5 for a representative HD. The $50 \%$ maximally stimulatory tyrosinase peptide concentration, representing the $K_{50}$ value, was $0.72 \mu \mathrm{g} / \mathrm{ml}$ for this particular HD. By contrast, CD8 + T cells specific for the CEF peptides were stimulated using a 200fold lower peptide concentration $\left(K_{50}=0.005 \mu \mathrm{g} / \mathrm{ml}\right)$. These peptide titrations studies indicate that tyrosinase-specific $\mathrm{CD} 8+\mathrm{T}$ cells exhibit low functional affinity for tyrosinase peptides, while CEF-specific CD8 + T cells display a high functional affinity for their cognate peptide.

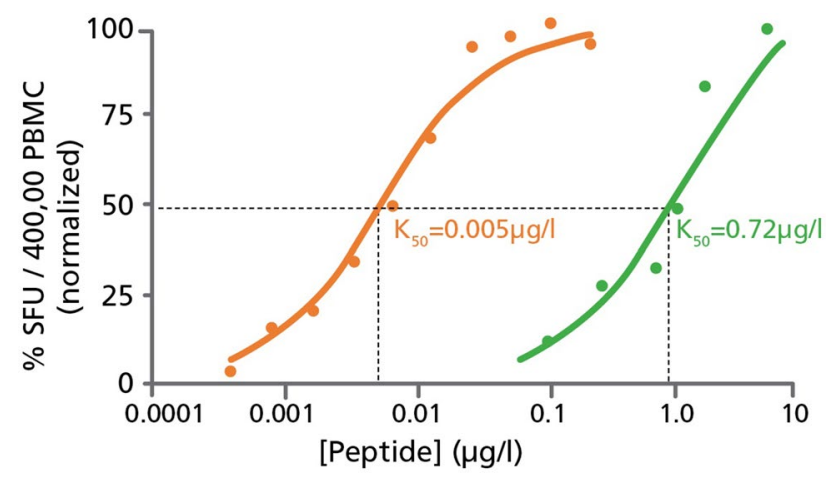

Fig. 5 Tyrosinase-specific $\mathrm{T}$ cells in healthy donors are of lowaffinity compared to CEF-specific T cells. Tyrosinase (green) and CEF (orange) peptides were serially diluted and used for testing at the specified concentrations using PBMC from HD \#17; who was shown to respond to both peptide pools (refer to Table 1). Taking the SFU counts elicited at $10 \mu \mathrm{g} / \mathrm{ml}$ of peptide as $100 \%$, the $\%$ response induced by the specified peptide concentrations is shown, and the peptide concentration that elicited $50 \%$ maximal stimulation was established as the $K_{50}$ value

\section{Discussion}

The present study was designed to address current technical challenges for ex vivo assessment of T cells in general, and MA-specific T cells in particular. This initial study serves as a baseline assessment using healthy donors, and building upon these data, eventually has application to characterizing $\mathrm{T}$ cell reactivity in melanoma patients. In contrast to previous studies that predominantly utilized select MA peptides in the context of specific HLA alleles and the flow cytometry-based multimer approach, we opted to utilize extensive peptide pools that systematically covered the entire amino acid sequence of the melanoma antigens; tyrosinase, MAGE-A3, Melan-A/Mart-1, gp100 and NY-ESO-1. The first surprising finding was that 23 of 40 healthy donors (58\%) displayed ex vivo reactivity to at least one of these MA antigens (Table 1). Eight of these HD responded to two or more MA. Specifically, two HD responded to two MA pools and another two HD responded to three MA pools. One HD responded to four MA, and the remaining three responded to all five MA peptide pools, respectively. NY-ESO-1 and gp100, while frequently recognized in combination with other MA, were not targeted in isolation. Overall, the MA-specific repertoire in HD appears to be diverse, and capable of recognizing each of five MA used in this study. Collectively, this observation justifies the use of all five of these peptide pools for comprehensive immune monitoring, and indicates that additional MA should be considered for assessment of $\mathrm{T}$ cell reactivity.

The present study also illustrates the feasibility of such a broad coverage of multiple antigens. As performed, using 250,000 PBMC per well and three replicates for each of the five MA plus the medium control, only 5.4 million PBMC were required to establish the breadth of the MAspecific repertoire from individual HD. Importantly, while this broad screening was performed using a single color IFN- $\gamma$ assay, the same number of PBMC could have been used to define the respective MA-specific T cell subpopulations using a 4-color four-color ImmunoSpot ${ }^{\circledR}$ assay. Thus, even after accounting for the limiting PBMC numbers that can be obtained from melanoma patients (e.g. 20 million PBMC), the number of MA peptide pools that could be readily tested can be increased to 25 when tested in triplicate, or 80 when tested as single replicates; which might well be permissible as hardly anyone runs samples in flow cytometry in replicates.

Using the ELISPOT platform for measurement of $\mathrm{T}$ cell responses against "foreign antigens", such as viruses, one can unambiguously identify donor cohorts that have, or have not, been exposed to a particular antigen. Such studies demonstrate that specific viral peptides, or peptide 
pools, elicit $\mathrm{T}$ cell-derived IFN- $\gamma$ production in excess of the medium background using PBMC from virus-exposed cohorts but not in virus-naïve individuals [22]. Confirming this notion, when we recently tested similar peptide pools that cover HCMV antigens, we found that IFN- $\gamma$ SFU were elicited only in CMV-exposed donors, but not in HCMV-negative individuals [23]. A similar experiment is detailed in Suppl. Fig. 1, which further illustrates this important point. In this study [23], and in the data presented in Suppl. Fig. 1, HCMV peptide pools that represent 11 distinct proteins encoded by of HCMV virus, each consisting of 15-mer peptides that span the entire length of the respective aa sequences in steps of 11 aa, were used for stimulation. These HCMV peptides were from the same manufacturer, JPT, and were synthesized and handled under identical experimental condition, including being used at an equivalent concentration as the MA peptides reported here. Each of the three HCMVinfected donors shown in Suppl. Fig. 1 (donors A, B and C) responded vigorously to most of the HCMV antigens/ peptide pools tested. In contrast, none of the three HCMV negative HD (donors $\mathrm{D}, \mathrm{E}$, and $\mathrm{F}$ ) responded to any of the 11 HCMV peptide pools with significant SFU numbers over the medium background. Thus, in a situation in which HCMV infection, and hence the development of an immune response, can be confirmed, the exquisite specificity of such peptide pools for detecting ex vivo-expanded memory T cells can easily be verified. However, since MA are ubiquitously expressed self-antigens, they may be more likely to induce immunologic tolerance than trigger clonal expansions and cytokine differentiation in healthy donors. Nevertheless, MA may be unique self-antigens since environmental factors such as sunburn, which promotes a proinflammatory environment, may enhance their immunogenicity and trigger initiation of an immune response.

The relatively high frequency of MA-specific CD8 + T cells detected amongst PBMC from HD was not anticipated. For example, under the $24-\mathrm{h}$ recall conditions (Table 1), tyrosinase-reactive T cells in HD \#7, \#9, \#17, and \#36 reached or exceeded $30 \mathrm{SFU}$ per 250,000 PBMC, which is a precursory frequency of $1: 8300$ or $0.008 \%$. In three additional HD (\#6, \#24 and \#31), MAGE-3A reactive cells were also present in this frequency range. While still at the detection limit of flow cytometry, the magnitude of these recall responses in an ELISPOT assay are typical of clonally expanded memory/effector $\mathrm{T}$ cell populations [24]. To this end, the frequency of MA-reactive CD8 + cells detected in these HD were in the range of clonally expanded memory cells, and were substantially greater than the $<1$ in 250,000 PBMC frequency anticipated in the naïve $\mathrm{T}$ cell compartment.

Using HLA-A $2 * 01$ tetramers, Melan-A ${ }_{(26-36)}$ peptidespecific CD $8+\mathrm{T}$ cells were detected at an atypically high frequency $(0.07 \%)$ in $\mathrm{HD}$, and these T cells were apparently naïve since they exhibited a CD45RA ${ }^{+}$surface phenotype [25]. As such a high frequency of naïve $T$ cells is unique, it is thought that these cells emerge through a particularly productive thymic selection process. As noted above, we have detected tyrosinase and Melan-A reactive $\mathrm{CD} 8+\mathrm{T}$ cells at frequencies around $0.01 \%$ following $24 \mathrm{~h}$ antigen stimulation directly ex vivo. After $72 \mathrm{~h}$ of antigen stimulation, 8 of $40 \mathrm{HD}$ responded to at least one of the MA at this relatively high, $>30$ SFU per 250,000 PBMC or $\sim 0.008 \%$, frequency range. Altogether, $58 \%$ of HD displayed a significant response to at least one of the five MA antigens used for testing.

In general, peptide-specific naïve $\mathrm{T}$ cells occur at very low frequencies; $<1$ per 250,000 PBMC. Additionally, naïve T cells do not secrete IFN- $\gamma$ within the first $24 \mathrm{~h}$ of antigen stimulation [10]. While naïve T cells are capable of extensive proliferation following antigen recognition, the 24-h incubation period of an ELISPOT assay is sufficient for only a single cell division cycle. Thus, detection of MA-reactive T cells capable of IFN- $\gamma$ production within a 24-h assay (Table 1; Fig. 1) signifies that these T cells have most likely undergone previous expansion and differentiation into Th1-type memory cells in vivo. Since the MA-specific T cells do not secrete GzB within the first $24 \mathrm{~h}$ of stimulation (Fig. 2b), these cells most likely represent resting (central) memory cells directly ex vivo. Following continued antigen stimulation, these cells go on to acquire expression of cytolytic granules and convert into GzB + effector CD8 + T cells capable of both cytolysis and IFN- $\gamma$ secretion. The observed behavioral pattern of these MA-reactive $\mathrm{T}$ cells is typical of resting CD $8+\mathrm{T}$ cells specific for virus antigens, including CEF peptidereactive $\mathrm{CD} 8+\mathrm{T}$ cells [21].

In this study we also identified MA-specific T cells from several HD that failed to secrete IFN- $\gamma$, along with GzB, after $24 \mathrm{~h}$ of antigen stimulation. However, these same donor cells yielded both IFN- $\gamma$ and GzB producing cells after $72 \mathrm{~h}$ of stimulation with MA (Table 1; Fig. 1). Such cells likely represent progeny of stem cell-like (SC) CD8 + memory $\mathrm{T}$ cells [9], which can engage in rapid proliferation and then differentiate into effector cells capable of both IFN- $\gamma$ and GzB expression following a longer $72 \mathrm{~h}$ antigen stimulation. Another possible explanation for the delayed cytokine secretion phenotype is that MA-specific T cells are naive at isolation. While self-antigen is likely to deplete the highaffinity end of the autoreactive T cell repertoire [16], T cells are positively selected on self-antigens and ongoing $\mathrm{T}$ cell receptor engagement is required for survival [26]. Therefore, the increased frequency of naïve MA-specific T cells could be attributed to an increased abundance of low-affinity, autoreactive $\mathrm{T}$ cells relative to those recognizing foreign antigens with high-affinity. 
Data consistent with the notion that self-reactive $\mathrm{T}$ cells in HD can possess a unique phenotype has also been reported for cytokeratin-18-specific CD8 + T cells [27]. Such cells have undergone extensive clonal expansion in vivo, but are functionally unresponsive and do not secrete cytokine in response to antigen encounter directly ex vivo. However, after antigen stimulation these cells regain their functionality. The MA-reactive T cells from HD that failed to secrete cytokines at $24 \mathrm{~h}$, but which became positive for cytokine secretion at $72 \mathrm{~h}$, therefore, might be the progeny of such clonally expanded autoreactive T cells. Overall, these findings suggest that in the context of comprehensive immune monitoring it is not advisable to restrict assessment of $\mathrm{T}$ cell function(s) to a single set time point following antigen stimulation. Instead, it would be preferable to evaluate $\mathrm{T}$ cell recall responses over extended time frames [28]. Importantly, this approach is technically feasible using the ELISPOT platform in spite of limiting PBMC acquired from patients. Furthermore, since ELISPOT is a non-destructive technique and $\mathrm{T}$ cells survive the assay largely unharmed, PBMC can theoretically be transferred from one assay into the next, facilitating testing of the same PBMC [29].

Initial efforts reported in the literature that sought to identify melanoma-specific $\mathrm{T}$ cells required over a week of in vitro expansion [5]. To avoid this in vitro expansion step, and to enable determination of MA-specific T cell precursor frequencies as they exist in vivo, we performed standard 24-h recall assays. This approach is commonly utilized to identify in vivo primed memory $\mathrm{T}$ cells. In an ELISPOT assay that does not require signal enhancers, the 24-h antigen-stimulation period is optimal for detecting peptideelicited cytokine production by CD8 + memory T cells [28]. Since resting memory CD $8+\mathrm{T}$ cells do not possess cytolytic granules immediately following antigen stimulation, but instead acquire this effector function within 3-4 days after antigen stimulation [21], we extended our in vitro culture to $72 \mathrm{~h}$ to determine whether the resting memory cells detected at $24 \mathrm{~h}$ could differentiate into cytolytic effectors. To enable segregation of such effector $\mathrm{T}$ cells into distinct sub-lineages, we also performed 4-color ImmunoSpot ${ }^{\circledR}$ analysis at the 72-h time point. The closer characterization of cytokine expression profiles by tyrosinase-specific $\mathrm{CD} 8+\mathrm{T}$ cells demonstrate that, in addition to terminal effector cells (TE: IL-2- IFN- $\gamma^{+}$, TNF- $\alpha^{+}$and $\mathrm{GzB}^{+}$), the polyfunctional (PF: IL- $2^{+}$, IFN- $\gamma^{+}, \mathrm{TNF}-\alpha^{+}$and $\mathrm{GzB}^{+}$) and stem-cell like (SC: IL- $2^{+}$, IFN- $\gamma^{-}$, TNF- $\alpha^{-}$and $\mathrm{GzB}^{-}$) lineages were also present within the MA-specific CD8 $+\mathrm{T}$ cell repertoires, with the latter stem cell-like subset prevailing (Fig. 4). The abundance of stem cell-like CD8 + T cells we observed serves as indirect evidence that tyrosinase-specific $\mathrm{CD} 8+\mathrm{T}$ cells in HD have not engaged in prolonged immune encounters in vivo. In contrast, in cancer patients, due to $\mathrm{T}$ cell exhaustion resulting from persistent antigen stimulation, senescent and dysfunctional tumor antigen-specific CD8 + T cells seem to prevail, with a concomitant reduction in the stem cell-like memory cell pool $[8,30]$.

Titration of the antigenic peptides permits determination of the functional affinity of antigen-specific $\mathrm{T}$ cells [16]. We found the tyrosinase-specific CD8 + T cells in HD to be of relatively low-affinity $\left(K_{50} \sim 1 \mu \mathrm{g} / \mathrm{ml}\right)$ compared to CEFspecific CD $8+\mathrm{T}$ cells $\left(K_{50} \sim 0.005 \mu \mathrm{g} / \mathrm{ml}\right)$. Unlike viral antigen-specific CD8 $+\mathrm{T}$ cells which recognized foreign peptide determinants, $\mathrm{T}$ cells recognizing self-antigens must somehow evade negative selection. To this end, our data suggest that the tyrosinase-specific CD $8+\mathrm{T}$ cells we detected in HD escaped negative selection due to their low affinity for this self-antigen. In contrast, $T$ cells bearing receptors that recognize tyrosinase peptides with high-affinity are likely to be purged from the repertoire through clonal deletion.

The data presented in this study also highlights the feasibility of evaluating the MA-specific T cell repertoire using a novel modification of the ImmunoSpot ${ }^{\circledR}$ technology. The sensitivity of the ELISPOT assay enables detection of low abundance $\mathrm{T}$ cells, far below the $<0.01 \%$ frequency range, which is not reliably achieved using a flow cytometry approach. The use of MA peptide pools also permitted parallel assessment of all potential antigenic determinants of the MA, which is not technically feasible using multimers. Studies of the activation kinetics also revealed an apparent naïve/anergic state of MA-specific T cells in vivo. Unlike flow cytometric assessment of cytokine production, which requires fixation and membrane permeabilization of the sample, cell viability is maintained during the ELISPOT and secondary assays can be performed without requiring additional cell material. Moreover, multi-color measurements of cytokine expression profiles using the 4-color ImmunoSpot ${ }^{\circledR}$ assay also permitted identification of the respective memory lineages and fractionation into stem cell-like, polyfunctional or terminal effectors cells. Finally, measurement of functional affinity was also deduced through peptide titration experiments, and was accomplished using fewer cells and requiring considerably less labor compared to flow cytometry. Importantly, all of the assays detailed in this report were accomplished using less than $20 \mathrm{ml}$ of blood per donor, and with relatively minor investment of investigator effort and cost. Furthermore, all of the reported ELISPOT assay variants can be readily validated and adapted for generating data in a regulated environment [11]. Thus, we believe, these novel extensions of the ImmunoSpot ${ }^{\circledR}$ technology will largely facilitate efforts to better understand tumor antigen-specific $\mathrm{T}$ cells in health and disease, including their mobilization for treatment of tumors.

Melanoma become immunogenic in the metastatic stage, and the $\mathrm{T}$ cell response to the malignant cells has been quite well-characterized. The $\mathrm{T}$ cells in melanoma patients target non-synonymous mutations on various proteins and/or 
recognize overexpressed non-mutated melanoma antigens $[2,31,32]$. However, it was not apparent prior to our findings that benign melanocytes were capable of triggering endogenous $\mathrm{T}$ cell responses against melanocyte autoantigens which are neither overexpressed, nor mutated, and as such qualify as "self-antigens". Our data draw attention to the existence of these melanocyte-specific CD8 + T memory cells in healthy human donors. Likely, this natural $\mathrm{T}$ cell autoreactivity gets primed during sunburns, when melanocyte activation occurs in the context of skin inflammation. At present, one can only speculate about the immunobiological significance of natural CD8 $+\mathrm{T}$ cell autoreactivity. One possible interpretation of this finding is that natural $\mathrm{T}$ cell autoreactivity to melanocyte antigens helps protects against development of melanoma. This notion is supported by ongoing studies, in which we found that 23 of 24 melanoma patients tested lacked MA reactivity that was demonstrated in this report using HD, while still exhibiting robust $\mathrm{T}$ cell reactivity against additional recall antigens (Anna Przybyla and Paul V. Lehmann-manuscript in preparation). A similar observation has been reported for breast cancer. Healthy women displayed high levels of spontaneous $\mathrm{T}$ cell autoreactivity to HER-2, while women with breast cancer were found to selectively lack such $\mathrm{T}$ cell responses [33]. One possible interpretation of such findings is that individuals lacking natural $\mathrm{T}$ cell autoreactivity to a given tumor are at increased risk of developing that cancer. However, there is an equally likely interpretation for the selective absence of natural $\mathrm{T}$ cell autoreactivity in cancer patients. Specifically, these pre-primed $\mathrm{T}$ cell populations may become "burned out" and undergo senescence in the face of ongoing antigen stimulation [34]. Such senescent T cells, while undetectable in an IFN- $\gamma$ recall assay, could even promote the growth of the tumor $[8,30]$. In either case, natural T cell autoreactivity targets autoantigens, and expression levels thereof, that are present on melanocytes. Once melanoma cells arise in the body, they offer a new set of target antigens for CD8 + T cell recognition, which can either be mutated or overexpressed melanoma antigens. The latter, though qualitatively unchanged relative to "self", become targeted because they are presented at much higher MHC-peptide density on tumor cells. When expressed at a sufficiently high copy number on transformed melanocytes, low-affinity autoreactive $\mathrm{T}$ cells will likely receive sufficient TCR stimulation that their activation threshold is exceeded. By contrast, these same lowaffinity autoreactive $\mathrm{T}$ cells would otherwise be ignorant of the same MHC-peptide combination when expressed at physiologic levels on normal melanocytes [35]. It is likely that checkpoint inhibitors may act on tumor-specific $\mathrm{T}$ cells that recognize mutated self-peptides, but it remains unclear whether such biologics also enhances the activity of pre-existing, natural $\mathrm{T}$ cell autoreactivity. Furthermore, the role of natural $\mathrm{CD} 8+\mathrm{T}$ cell reactivity and prevention of melanoma in HD is presently unknown. Whether these cells are inactive in melanoma patients, or if they contribute to promoting the immunosuppressive tumor micro-environment after undergoing senescence are also key questions that will require further study.

Acknowledgements The authors wish to acknowledge Dr. Greg Kirchenbaum for his critical feedback and review of the manuscript.

Author contributions AP contributed the data for Figs. 1, 2, 3 and 5, as well as Table 1. The experiments shown in Fig. 4 were performed by DRR. The data shown in Suppl. Fig. 1 were generated by TZ and RL. AM and PVL designed and supervised the study.

Funding This study was conducted in Research and Development Department of Cellular Technology Limited (CTL) and was funded from the research budget of CTL. The study was also supported by the National Centre for Research and Development (Warsaw), Project Innomed: Personalization of melanoma therapeutic vaccination. Innomed/I/6/NCBR/2014.

\section{Compliance with ethical standards}

Conflict of interest Paul V. Lehmann is founder and CEO of CTL, a company that specializes on immune monitoring via ELISPOT. Ting Zhang, Ruliang Li., and Diana R. Roen are CTL employees. Anna Przybyla and Andrzej Mackiewicz declare that they have no conflict of interest.

Informed consent The PBMC of healthy human donors used in this study were obtained from CTL's ePBMC bank. (http://www.epbmc .immunospot.comCTL, Cleveland, OH, USA). These cells were isolated from blood purchased from Hemacare (Van Nuys, CA, USA) under Hemacare's IRB. Hemacare obtained the informed consents from healthy blood donors.

Research involving human and/or animal participants This article does not contain any studies involving patients or experimental animals.

Ethical approval PBMC from healthy human donors used for this study were collected with IRB approval.

OpenAccess This article is distributed under the terms of the Creative Commons Attribution 4.0 International License (http://creativeco mmons.org/licenses/by/4.0/), which permits unrestricted use, distribution, and reproduction in any medium, provided you give appropriate credit to the original author(s) and the source, provide a link to the Creative Commons license, and indicate if changes were made.

\section{References}

1. Drake CG, Lipson EJ, Brahmer JR (2014) Breathing new life into immunotherapy: review of melanoma, lung and kidney cancer. Nat Rev Clin Oncol 11:24-37. https://doi.org/10.1038/nrcli nonc. 2013.208

2. Lee PP, Yee C, Savage PA et al (1999) Characterization of circulating $\mathrm{T}$ cells specific for tumor-associated antigens in melanoma patients. Nat Med 5:677-685. https://doi.org/10.1038/9525 
3. Nagorsen D, Scheibenbogen C, Marincola FM, Letsch A, Keilholz U (2003) Natural T cell immunity against cancer. Clin Cancer Res 9:4296-4303

4. Zinkernagel RM, Ehl S, Aichele P, Oehen S, Kundig T, Hengartner H (1997) Antigen localisation regulates immune responses in a dose- and time-dependent fashion: a geographical view of immune reactivity. Immunol Rev 156:199-209

5. Romero P, Cerottini JC, Speiser DE (2006) The human T cell response to melanoma antigens. Adv Immunol 92:187-224. https ://doi.org/10.1016/s0065-2776(06)92005-7

6. Parmiani G (2001) Melanoma antigens and their recognition by T cells. Keio J Med 50:86-90

7. Zippelius A, Batard P, Rubio-Godoy V et al (2004) Effector function of human tumor-specific CD8 T cells in melanoma lesions: a state of local functional tolerance. Cancer Res 64:2865-2873

8. Apetoh L, Smyth MJ, Drake CG et al (2015) Consensus nomenclature for CD8(+) T cell phenotypes in cancer. Oncoimmunology 4:e998538. https://doi.org/10.1080/2162402x.2014.998538

9. Lugli E, Dominguez MH, Gattinoni L et al (2013) Superior T memory stem cell persistence supports long-lived T cell memory. J Clin Investig 123:594-599. https://doi.org/10.1172/jci66327

10. Macatonia SE, Hosken NA, Litton M et al (1995) Dendritic cells produce IL-12 and direct the development of Th1 cells from naive CD4 + T cells. J Immunol (Baltimore Md 1950) 154:5071-5079

11. Tary-Lehmann M, Hamm CD, Lehmann PV (2008) Validating reference samples for comparison in a regulated ELISPOT assay. In: Prabhakar U, Kelley M (eds) Validation of cell-based assays in the GLP setting: a practical guide. Wiley, Hoboken, New Jersey, United States, p 127

12. Altman JD, Moss PA, Goulder PJ, Barouch DH, McHeyzer-Williams MG, Bell JI, McMichael AJ, Davis MM (1996) Phenotypic analysis of antigen-specific T lymphocytes, vol 274. Science, New York, pp 94-96

13. Zhang W, Moldovan I, Targoni OS, Subbramanian RA, Lehmann PV (2012) How much of virus-specific CD8 T cell reactivity is detected with a peptide pool when compared to individual peptides? Viruses 4:2636-49. https://doi.org/10.3390/v4112636

14. Hanson J, Roen DR, Lehmann PV (2018) Four color ImmunoSpot ${ }^{\circledR}$ assays for identification of effector T-cell lineages. Methods Mol Biol (Clifton NJ) 1808:51-62. https://doi. org/10.1007/978-1-4939-8567-8_5

15. Karulin AY, Hesse MD, Tary-Lehmann M, Lehmann PV (2000) Single-cytokine-producing CD4 memory cells predominate in type 1 and type 2 immunity. J Immunol (Baltimore Md 1950) 164:1862-1872

16. Targoni OS, Lehmann PV (1998) Endogenous myelin basic protein inactivates the high avidity $\mathrm{T}$ cell repertoire. J Exp Med 187:2055-2063

17. Ramachandran H, Laux J, Moldovan I, Caspell R, Lehmann PV, Subbramanian RA (2012) Optimal thawing of cryopreserved peripheral blood mononuclear cells for use in high-throughput human immune monitoring studies. Cells 1:313-324. https://doi. org/10.3390/cells1030313

18. Currier JR, Kuta EG, Turk E, Earhart LB, Loomis-Price L, Janetzki S, Ferrari G, Birx DL, Cox JH (2002) A panel of MHC class I restricted viral peptides for use as a quality control for vaccine trial ELISPOT assays. J Immunol Methods 260:157-172

19. Schiller A, Zhang T, Li R, Duechting A, Sundararaman S, Przybyla A, Kuerten S, Lehmann PV (2017) A positive control for detection of functional CD4 T cells in PBMC: the CPI pool. Cells. https://doi.org/10.3390/cells6040047

20. Karulin AY, Caspell R, Dittrich M, Lehmann PV (2015) Normal distribution of CD8 + T-cell-derived ELISPOT counts within replicates justifies the reliance on parametric statistics for identifying positive responses. Cells 4:96-111. https://doi.org/10.3390/ cells4010096

21. Nowacki TM, Kuerten S, Zhang W, Shive CL, Kreher CR, Boehm BO, Lehmann PV, Tary-Lehmann M (2007) Granzyme B production distinguishes recently activated CD8(+) memory cells from resting memory cells. Cell Immunol 247:36-48. https://doi. org/10.1016/j.cellimm.2007.07.004

22. Kuerten S, Nowacki TM, Kleen TO, Asaad RJ, Lehmann PV, Tary-Lehmann M (2008) Dissociated production of perforin, granzyme B, and IFN-gamma by HIV-specific CD8(+) cells in HIV infection. AIDS Res Hum Retrovir 24:62-71. https://doi. org/10.1089/aid.2007.0125

23. Terlutter F, Caspell R, Nowacki TM, Lehmann A, Li R, Zhang T, Przybyla A, Kuerten S, Lehmann PV (2018) Direct detection of T- and B-memory lymphocytes by ImmunoSpot ${ }^{\circledR}$ assays reveals HCMV exposure that serum antibodies fail to identify. Cells. https ://doi.org/10.3390/cells7050045

24. Moldovan I, Targoni O, Zhang W, Sundararaman S, Lehmann PV (2016) How frequently are predicted peptides actually recognized by CD8 cells? Cancer Immunol Immunother 65:847-855. https:// doi.org/10.1007/s00262-016-1840-7

25. Pittet MJ, Valmori D, Dunbar PR et al (1999) High frequencies of naive Melan-A/MART-1-specific CD8(+) T cells in a large proportion of human histocompatibility leukocyte antigen (HLA)-A2 individuals. J Exp Med 190:705-715

26. Starr TK, Jameson SC, Hogquist KA (2003) Positive and negative selection of T cells. Annu Rev Immunol 21:139-176. https://doi. org/10.1146/annurev.immunol.21.120601.141107

27. Walter S, Bioley G, Buhring $\mathrm{HJ}$ et al (2005) High frequencies of functionally impaired cytokeratin 18-specific CD8 + T cells in healthy HLA-A2 + donors. Eur J Immunol 35:2876-2885. https ://doi.org/10.1002/eji.200526207

28. Duechting A, Przybyla A, Kuerten S, Lehmann PV (2017) Delayed activation kinetics of Th2- and Th17 cells compared to Th1 cells. Cells. https://doi.org/10.3390/cells6030029

29. Ott PA, Dittrich MT, Herzog BA et al (2004) T cells recognize multiple GAD65 and proinsulin epitopes in human type 1 diabetes, suggesting determinant spreading. J Clin Immunol 24:327339. https://doi.org/10.1023/b:Joci.0000029120.77824.41

30. Naugler WE, Karin M (2008) The wolf in sheep's clothing: the role of interleukin-6 in immunity, inflammation and cancer. Trends Mol Med 14:109-119. https://doi.org/10.1016/j.molme d.2007.12.007

31. Lennerz V, Fatho M, Gentilini C, Frye RA, Lifke A, Ferel D, Wolfel C, Huber C, Wolfel T (2005) The response of autologous $\mathrm{T}$ cells to a human melanoma is dominated by mutated neoantigens. Proc Natl Acad Sci USA 102:16013-16018. https://doi. org/10.1073/pnas.0500090102

32. Ott PA, Hu Z, Keskin DB et al (2017) An immunogenic personal neoantigen vaccine for patients with melanoma. Nature 547:217221. https://doi.org/10.1038/nature22991

33. Datta J, Rosemblit C, Berk E et al (2015) Progressive loss of anti-HER2 CD4(+) T-helper type 1 response in breast tumorigenesis and the potential for immune restoration. Oncoimmunology 4:e1022301. https://doi.org/10.1080/2162402x.2015.1022301

34. Wherry EJ, Kurachi M (2015) Molecular and cellular insights into T cell exhaustion. Nat Rev Immunol 15:486-499. https://doi. org/10.1038/nri3862

35. Lehmann PV, Targoni OS, Forsthuber TG (1998) Shifting T-cell activation thresholds in autoimmunity and determinant spreading. Immunol Rev 164:53-61 